\title{
Evidence from epidemic appraisals in Nigeria
}

\begin{abstract}
Authors:
Idoteyin O. Ezirim ${ }^{1}$

Kayode Ogungbemi ${ }^{1}$

Uduak Daniel $^{1}$

James Blanchard ${ }^{2}$

Onoriode Ezire ${ }^{3}$

\section{Affiliations: \\ ${ }^{1}$ National Agency for the Control of AIDS (NACA), Nigeria \\ ${ }^{2}$ University of Manitoba, Canada}

${ }^{3}$ Society for Family Health (SFH), Nigeria

\section{Correspondence to:}

Idoteyin Ezirim

\section{Email:}

send2teyin@yahoo.com

\section{Postal address:}

PO Box 2370, Garki Abuja,

Nigeria

\section{Dates:}

Received: 08 May 2015

Accepted: 11 Sept. 2015

Published: 12 Oct. 2015

How to cite this article: Ezirim, I.O., Ogungbemi, K., Daniel U, Blanchard, J. \& Ezire, O., 2015, 'Evidence from epidemic appraisals in Nigeria', African Evaluation Journal 3(2), Art. \#153, 8 pages. http://dx.doi. org/10.4102/aej.v3i2.153

\section{Copyright:}

(C) 2015. The Authors. Licensee: AOSIS OpenJournals. This work is licensed under the Creative Commons Attribution License.
\end{abstract}

\section{Read online:}

Although HIV prevalence has increased in most-at-risk populations (MARPs) across Nigeria, effective programming was difficult because Nigeria lacked information for prevention programmes to target interventions that maximise coverage and cost effectiveness. Epidemic appraisals (EA) were conducted in eight states to provide evidence for the planning, implementation and co-ordination of prevention interventions. Component 1: Mapping determined the size, typology and locations of MARPs. Component 2: Venue profiling identified and profiled venues where general populations engaged in high-risk behaviours. Component 3: Rural appraisals provided insights into risk behaviours and sexual networking in villages. States used mapping results to prioritise areas with a MARP coverage of $70 \%-80 \%$ and then scale up interventions for non-brothel-based female sex workers (FSWs) instead of focusing on brothel-based FSWs. The eight states prioritised funding for the high-coverage areas to ensure a minimum coverage level of $70 \%-80 \%$ of MARPs was reached. The refocused resources led to cost efficiencies. Applying venue profiling results, six states implemented interventions at bars and night clubs - previously not covered. States also maximised intervention coverage for high-risk general populations; this led to the use of resources for general population interventions in a focused way rather than across an entire state. States focused on condom programmes in rural areas. EA results provided the evidence for focusing interventions for high MARP coverage as well as for high-risk general populations. The states applied the results and rapidly refocused their interventions, increasing the likelihood of having an impact on HIV transmission in those states. Nigeria is now implementing EAs in the remaining 29 states to effect national-level impact.

\section{Introduction Problem statement}

Some of the challenges for HIV-prevention programming in Nigeria have included a lack of adequate evidence for programme planning, gaps in the knowledge of what is driving the HIV epidemic in different regions of Nigeria, and inadequate knowledge of the geographic distribution of key populations. These challenges hinder the targeting of HIV-prevention resources where they will have the highest impact. And, although evidence shows that HIV prevalence is increasing across most-at-risk populations (MARP) in Nigeria, programming for these populations remains difficult because of inadequate information on strategies that maximise the coverage and cost effectiveness of HIV interventions.

Nigeria is the most populous country in Africa. The country is organised into 36 states and the Federal Capital Territory (FCT). For administrative purposes, the states are further divided into 774 local government areas (LGAs). Nigeria's HIV prevalence is highest amongst MARPs (female sex workers (FSW), injecting drug users (IDU), and men who have sex with men (MSM), particularly those who have multiple sexual partners.) A female sex worker is defined as a woman who exchanges sex for money or other items of value, primarily with men (National Agency for the Control of AIDS (NACA) 2014:10)

Nigeria conducted integrated biological and behavioural surveillance (IBBS) surveys in 2007 and 2010 amongst MARPs in 6 and 10 Nigerian states, respectively (FMOH [2007] 2010:26-67, 14-27). Survey results showed high HIV prevalence in all three MARP groups, particularly those engaged in high-risk sexual behaviours, as shown in Table 1. The average HIV prevalence in brothel-based FSWs was the highest: $37.4 \%$ in 2007 and $27.4 \%$ in 2010. Prevalence was $30.2 \%$ and $21.7 \%$ amongst non-brothel-based FSWs, $13.6 \%$ and $17.2 \%$ amongst MSMs, and 5.6\% and $4.2 \%$ amongst IDUs in 2007 and 2010, respectively. Whilst MARPs account for only an estimated $3.4 \%$ of the national population, they account for as much as $40 \%$ of new HIV infections (NACA, GARPR 2012). 
TABLE 1: Percentage of HIV prevalence amongst most-at-risk populations in Nigeria, 2007 and 2010.

\begin{tabular}{lcccc}
\hline Year & Brothel-based sex workers & Non-brothel-based sex workers & MSM & IDU \\
\hline 2007 & 37.4 & 30.2 & 13.6 & 5.6 \\
2010 & 27.4 & 21.7 & 17.2 & 4.2 \\
\hline
\end{tabular}

Source: National Agency for the Control of AIDS (NACA), 2013, 'HIV epidemic appraisals in Nigeria: Evidence for prevention programme planning and implementation. Data from the first eight states', viewed 15 March 2015, from http://www.sbccvch.naca.gov.ng/sites/ default/files/Local\%20Epidemic\%20Appraisal_glossy\%20report.pdf

The Government of Nigeria declared HIV prevention a national health sector and development priority with a national goal to intensify, scale up and accelerate prevention efforts. Previous assessments of the HIV epidemic in Nigeria (FMOH [2008] 2010:18-53, 14-50) showed a mixed epidemic in many regions. It also revealed that the epidemic is driven by HIV transmission both within networks involving MARPs and also within segments of the general population with multiple partners and/or belonging to sexual networks that facilitate the spread of HIV. Evidence from these assessments also shows that Nigeria's HIV epidemic appeared to be highly heterogeneous with respect to its geographic distribution.

Although data from routine surveillance (HIV sentinel survey amongst antenatal care attendees), behavioural or biological surveys (IBBSS, National HIV\&AIDS and Reproductive Health Survey [NARHS]), and routine programme data provided information on the HIV epidemic, there were still gaps in information for HIV prevention activities for MARPs at the LGA (district) level. Nigeria also needed to assess the nature of other high-risk sexual networks, the extent to which they overlapped with MARPs, and key aspects of the behaviour driving HIV transmission in the general population. Finally, Nigeria needed to optimise intervention coverage to these populations, maximise its impact by allocating resources efficiently within a state, and select the right intervention strategy in order for HIV-prevention programmes to be effective and efficient at local levels. The specific information gaps included:

- The identification and selection of geographical areas to target HIV-prevention programmes to maximise the efficiency of reach, coverage and impact.

- Knowing how best to estimate the population sizes, and to determine the operational typology of MARPs in each location and their implication for intervention strategies.

The findings from epidemic appraisals (EA), a methodology for identifying areas of high density and high risk, play a very important role in planning an effective and efficient HIVprevention strategy because they provide evidence for focusing on high coverage areas for MARP-targeted interventions. Nigeria conducted eight state-level EAs, adapting the methodology used in India (India Health Action Trust 2010). Themapin Figure1 shows theeightstates assessed:FCT(Abuja), Anambra, Benue, Cross River, Gombe, Lagos, Nasarawa, and Ondo. To address Nigeria's mixed epidemic, one key difference in the EA methodology used in Nigeria was the assessment of other high-risk networks in the general population (NACA 2013) whereas in the EAs in India only hotspots were mapped.

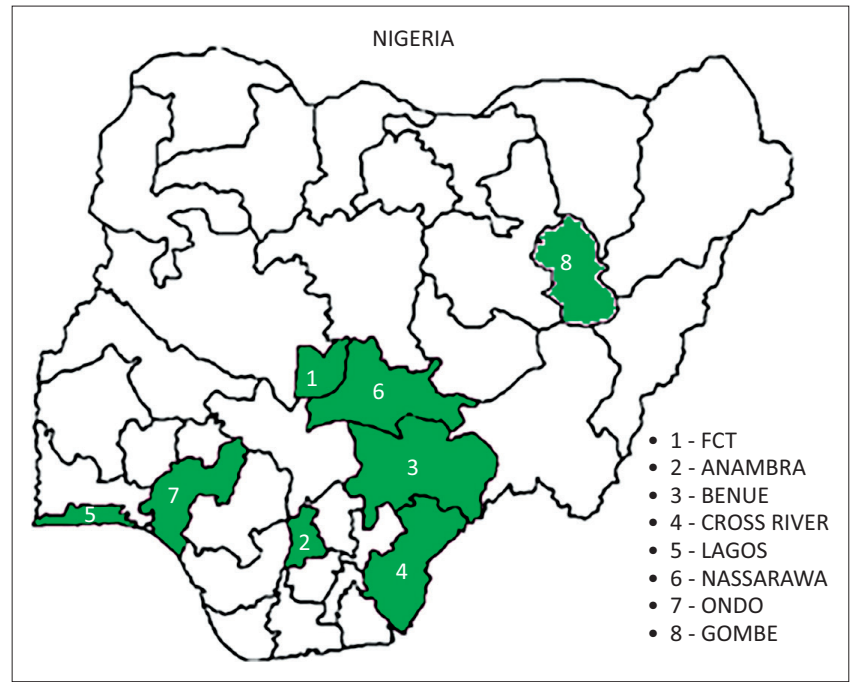

Source: National Agency for the Control of AIDS (NACA), 2013, 'HIV epidemic appraisals in Nigeria: Evidence for prevention programme planning and implementation. Data from the first eight states', viewed 15 March 2015, from http://www.sbccvch.naca.gov.ng/sites/ default/files/Local\%20Epidemic\%20Appraisal_glossy\%20report.pdf

FIGURE 1: States included in the epidemic appraisal.

This article describes how the EA results were used for planning and targeting HIV prevention programmes in eight Nigerian states. (The full Nigeria EA results have been reported elsewhere (NACA 2013).)

The team focused on these four objectives:

1. To describe how the findings from the eight state-level EAs could form the framework for planning, implementing and co-ordinating MARP interventions in Nigeria.

2. To demonstrate the pivotal and central nature of the EA results to MARP prevention programming.

3. To show how the EA-generated evidence is being used by all stakeholders in Nigeria for allocating resources efficiently and prioritising target populations.

4. To illustrate how EAs can be used to achieve optimal coverage of prevention services for the high-risk general population.

\section{Literature review}

\section{The epidemic appraisal}

The EA is a methodology countries can use to understand HIV epidemic typologies and drivers (Blanchard 2012:15). The University of Manitoba, in collaboration with the World Bank, provided technical support to India, Kenya, Pakistan, and Nigeria to conduct EAs (Blanchard 2012:15).

The use of EAs to conduct geographic mapping of key populations was first done in India. EAs were used in India to map hotspots in order to determine locations and to estimate the population sizes of key populations. These data were triangulated with available data from routine surveillance (HIV sentinel survey amongst antenatal care attendees) as well as behavioural/biological surveys and routine programme data (India Health Action Trust 2010). The results from the India EAs were used to plan service delivery at the LGA level and to prioritise resource allocations based 
on population size estimates (UNAIDS Reference Group 2013:5-7). Likewise in Pakistan, population size estimates were derived from EAs conducted in 2011 amongst IDUs, hijra (transgender individuals), male sex workers (MSW) and FSWs. In both countries, cities were prioritised according to key population size. Programme costs per person were used to estimate the cost of delivering services to $80 \%$ of these key population members, the coverage target to reduce HIV prevalence (Emmanuel et al. 2013).

India implemented HIV-prevention interventions, targeting $80 \%$ of key populations in the prioritised cities. An impact evaluation of these programmes has shown a reversal of the epidemic amongst both FSWs and their clients and the general population (Emmanuel et al. 2013), indicating that prevention programmes targeting the correct populations at high coverage levels can have an impact on the epidemic.

EAs have also been conducted in Kenya and the findings used for a geographic prioritisation approach to produce cost efficiencies. This in turn resulted in an increased impact without increasing the amount spent on the programme (UNAIDS Reference Group 2013:5).

As a general rule, when applying a Know your epidemic and know your response approach to a mixed epidemic, the prevention priority objective is to achieve high coverage of targeted interventions for MARPs, focusing on locations where there is a substantial overlap with general population networks (Wilson \& Halperin 2008). The IBBSS results confirmed the existence of various typologies of MARPs in Nigeria (FMOH [2007] 2010:26-67, 14-27). Gaps in the available information on Nigeria's epidemic included lack of:

1. specifics on the geographic distribution of key populations, which has hindered the targeting of HIV prevention to them;

2. specifics on how to target the general population engaging in high-risk behaviour;

3. information to guide resource allocation for maximum population-level impact;

4. knowledge about the typology and size of key populations and the implications for intervention strategies and scaling-up coverage.

\section{Nigeria's adaptation of the epidemic appraisals methodology, and the results}

To address the information gaps, NACA adapted the EA methodology to assess casual sexual networks in urban areas and the general population in rural areas in addition to mapping key populations in hotspots.

The Nigeria EA results from the eight states detailed the size estimates for MARPs by LGA, the typology of MARPs in these states, and the venues in the states where high-risk sexual activities took place. The size estimations from the EAs provided direction for optimal programme coverage. LGAs with both the highest population and density of MARPs were prioritised and targeted for service provision to ensure that $70 \%-80 \%$ of MARPs are reached with interventions. Following the LGA prioritisation, partners implementing HIV prevention interventions were assigned to work in the prioritised LGAs in a consultative process with each State Agency for the Control of AIDS (SACA).

In addition, size estimations from the EAs were used with the cost per unit of intervention to estimate the cost to deliver services at $70 \%-80 \%$ coverage level in the prioritised LGAs. NACA in collaboration with donor agencies examined the total resource envelope available for MARP interventions in Nigeria and the unit cost of providing interventions to determine resource allocation to the prioritised LGAs. This method ensured that resources were allocated in a costefficient manner to achieve the highest population impact rather than applying available resources to provide services for all MARPs in the state.

\section{Research method and design Setting}

These EAs were conducted in eight Nigerian states in urban and rural areas, as described above in the Literature Review section.

\section{Design}

The EA was conducted using a mixed method design involving both quantitative and qualitative data collection. The protocols for all three components were approved by the National Health Research Ethics Committee of the State Ministry of Health (SMOH) in each of the eight states.

\section{Procedure}

This section summarises the sample size selection and data collection methods for each component of the EA methodology.

\section{Mapping most-at-risk populations}

Mapping was used to determine the size of the MARP groups, their typology and their locations. The information on size and location is used to prioritise the targeting of interventions where there is coverage of $70 \%-80 \%$ of the MARPs. This information, along with cost per unit of intervention is used to determine the resources needed to achieve the desired coverage in the selected locations and guide allocation of resources. Information on the MARP typology is used to tailor the programme approach (coverage and type of intervention). The cross-sectional study design enabled the survey to be conducted rapidly, which was important because of the mobile nature of the MARP groups.

In all of the states except FCT and Lagos, major LGAs and towns in the state were selected as study areas. The towns selected were considered to be the most important in terms of presence and size of key populations. Researchers used up-to-date geographical maps to divide the study area 
into smaller units (referred to as 'zones') based on one of the following factors considered applicable to the state: an existing geographical division, physical landmarks, or an existing administrative division. In Lagos and FCT, the whole state was divided into zones, as there are no welldefined towns in these states (with Emmanuel et al. 2012).

Mapping involved two sequential levels of data collection. In level 1 mapping, secondary key informants such as taxi drivers, bar workers, brothel owners, security men and petty shop owners were interviewed to identify locations where members of these key populations congregate. In level 2 mapping, the locations identified in the level 1 mapping were visited and key population members (primary key informants) at those locations were interviewed to obtain more detailed information. This information included the characterisation and size estimate of the MARPs in that location (with Emmanuel et al. 2012). The characterisation information collected during the level 2 mapping included the type of spot operating at the location, the time of operation when solicitation takes place and the type of key population that can be found at the location.

\section{Venue profiling (urban)}

Venue profiling is used to identify and profile venues where segments of the general population engage in high-risk behaviour. The information ensures that HIVprevention services are focused on these venues to reach a large segment of the general population seeking casual sex in urban areas. The probability-proportional-to-size (PPS) sampling method was used for the probability of selecting a sampling unit (in this case, a zone) proportional to the size of the population (implementation details are available in Shajy et al. 2012b).

Venue profiling includes four stages: selection of zones, venue listing, consolidation of lists and profiling venues:

1. In stage one, selection of zones, the geographic study area was selected. The PPS sampling selected a representative sample of the zones used during the geographic mapping of MARPs.

2. For venue listing (stage two), field workers visited each zone/subzone and collected information from secondary key informants about venues where people congregate for social activities. These venues were compiled as the Venue list.

3. In stage three, the consolidation of lists, each Venue list was merged with the list of hotspots generated earlier during the MARP mapping for that zone/subzone. The merged list, the Master list of venues, contains a complete list of the venues to be profiled.

4. In stage four, using the Master list of venues, the field workers visited all the listed venues and interviewed primary key informants.

\section{Rural appraisal methodology}

The rural appraisal provides insight into high-risk behaviours and sexual networking that occur in villages and small towns. Programmes can then design strategies for rural areas aimed at promoting behaviour change for activities with high sexual risk. The rural appraisal study design is a bridge between formal surveys and research methods such as in-depth interviews, focus group and observational studies. This approach is advantageous because less time is taken to produce results, the cost of surveying is low, and it reduces social desirability bias in HIV-related behavioural surveys in resource-poor settings (Shajy et al. 2012a). Rural appraisal comprises two components: the rapid assessment (RA) and the polling booth survey (PBS).

\section{Rapid assessment}

Each state was divided into four geographic zones with distinct and contiguous LGAs. Using a comprehensive village list, the 20 most populous villages in each geographic zone were then selected, which resulted in a target sample size of 80 villages in each state. In each village, five key informants who were knowledgeable and had reliable information about their village were selected. Each key informant was interviewed and the information collected was recorded using the RA sheet, a tool used for data collation and triangulation. This tool allowed all the responses to questions from the five key informants to be recorded on one sheet (for details, see Shajy et al. 2012a).

\section{Polling booth survey}

The PBS comprises five steps:

1. Step 1: Identification of demographic groups: Demographic groups were selected as described in Table 2.

2. Step 2: Selection of villages: Using the complete list of villages obtained in the RA, the 17 most populous villages were selected for a total of 68 villages.

3. Step 3: Segmentation of the village: Each selected village was segmented geographically and then the respondents for each of the demographic groups were sampled.

4. Step 4: Household listing: Each field team was assigned to a segment and began the household listing process by randomly selecting a house where they enlisted an eligible participant - that is, someone who met the demographic group criteria. The teams continued in a clockwise direction to the next household to recruit an eligible participant and so on until 12 eligible participants were assembled, after which they accompanied the team to the PBS location.

5. Step 5: Conducting the PBS: Each respondent used a private polling booth to answer a series of closed-ended

TABLE 2: Demographic groups for polling booth survey sampling.

\begin{tabular}{lll}
\hline Variables & Age range & \\
\hline Married & & \\
Female & $15-29$ years & $30-49$ years \\
Male & $15-29$ years & $30-49$ years \\
Unmarried & \\
Female & $15-24$ years & $25-34$ years \\
Male & $15-24$ years & $25-34$ years \\
\hline
\end{tabular}

Source: National Agency for the Control of AIDS (NACA), 2013, 'HIV epidemic appraisals in Nigeria: Evidence for prevention programme planning and implementation. Data from the first eight states', viewed 15 March 2015, from http://www.sbccvch.naca.gov.ng/sites/ default/files/Local\%20Epidemic\%20Appraisal_glossy\%20report.pdf 
questions. To reply, the respondents used coloured cards designated to represent the following possible responses: Yes, No, and Not applicable, as well as 'no card' in cases where the respondent chose not to respond.

\section{Analyses}

MS Access 2007 databases were used for the initial analysis of the mapping and venue profiling data; these data were then exported to MS Excel and further analysis was conducted on collated RA and PBS data. (See details in Emmanuel et al. 2012, Shajy et al. 2012a ; Shajy et al. 2012b).

\section{Discussion}

\section{Outline of the results}

EAs provided information on the geographic distribution and typology of key populations as well as venues for highrisk sexual behaviour. Across the eight states, hotspots were identified, the size of key populations by type of hotspot was estimated and venues for casual sexual networking were profiled.

Several key findings (KF) emerged from the EAs that guided the HIV-prevention response. These are summarised below, along with the programme actions taken at the national and state levels.

\section{Programming for the most-at-risk populations}

KF1: Non-brothel-based FSWs were not being covered by interventions.

The EAs identified that a large proportion of FSWs were not based in brothels. At that time, the majority of HIVprevention programmes in Nigeria focused only on brothelbased FSWs, which would have yielded coverage of only $16 \%$ of all urban FSWs across the eight states.

Action taken: The National Prevention Technical Working Group (NPTWG) adopted a strategy to expand prevention programmes to other types of FSWs, particularly those working in bars and nightclubs (28\% of FSWs) and in hotels and lodges (32\% of FSWs) (NACA 2013).

KF2: The eight states needed to focus on key LGAs with high concentrations of FSWs to achieve optimal programme coverage.
At the state level, mapping found large differences in the size and density of FSW populations amongst the LGAs. For example, whereas all five LGAs in FCT were mapped, the EA results showed that three LGAs accounted for $90 \%$ of the FSW hotspots and estimates.

Action taken: The scale-up of FSW interventions was refocused on those LGAs with both the highest population and the highest density of FSWs to achieve at least $80 \%$ coverage. Services were targeted first for the prioritised LGAs identified from the EAs.

KF3: MARP programme resources were being used in a less cost-efficient model.

The limited available resources meant that the approach of providing services equally to all the MARPs in a state would not necessarily have the desired impact on the HIV epidemic.

Action taken: NACA, in collaboration with donor agencies, applied the EA size estimations and the unit cost of providing interventions to determining the resource allocations needed for service delivery in the prioritised LGAs in each state.

KF4: The EA hotspot lists allowed NACA to gain coverage and resource efficiencies through improving the model of service delivery for FSWs.

The hotspot maps informed the adoption of the cluster model of service delivery for FSWs in Nigeria.

Action taken: The SACAs, in consultation with implementing partners, clustered hotspots and linked these hotspots to nearby health facilities providing the MARPfriendly services. The use of this model to ensure that FSWs received the complete package of combination prevention (behavioural, biomedical and structural) led to the adoption of the cluster model of service delivery in the National Guidelines for Implementing HIV Prevention Programmes for FSW in Nigeria in 2014. An example of a cluster worksheet used in Cross River state for service delivery to FSW hotspots is presented in Table 3.

\section{Programming for high-priority urban general populations}

KF5: Specific venues provide an efficient target for focused HIV-prevention programmes for general populations.

TABLE 3: Cluster a worksheet for cross river state (LGA = Calabar Municipality).

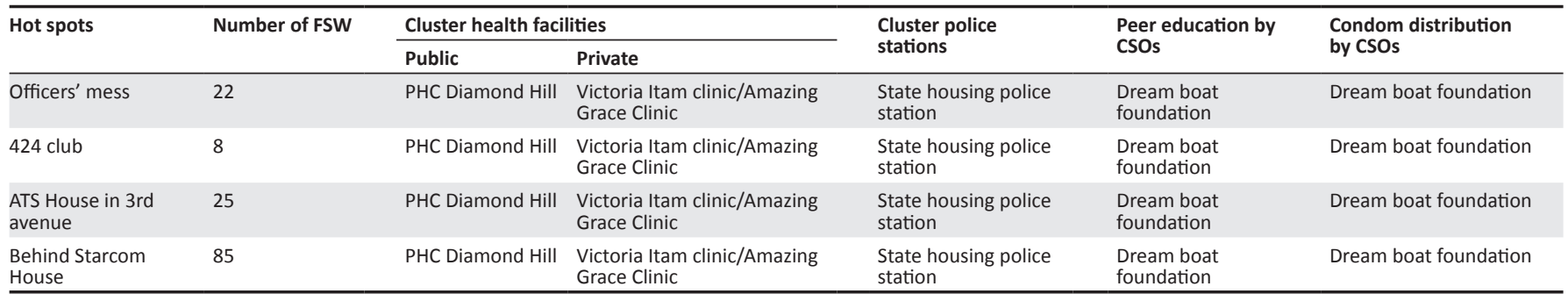


Venue profiling provided information about the number and types of venue used by the general population for meeting new sexual partners, thereby providing information about where to focus interventions. For example, $73 \%$ of the venues in Cross River state were found to be high risk - that is, they facilitated finding new sexual partners. Approximately 34 patrons visited each of the high-risk venues in Cross River on a typical day, with approximately $35 \%$ of these patrons seeking sexual partners at these venues.

Action taken: Refocusing HIV general prevention efforts on key venues maximised coverage of higher-risk individuals in the states.

KF6: Bars, nightclubs, hotels and lodges are the most important venues to target.

Bars and night clubs were most commonly associated with sexual networking in six of the states, indicating the need to prioritise these venues.

Action taken: In six states, bars and night clubs were targeted for intervention.

KF7: Many of the venues where the general population met new casual sexual partners also have FSWs and their clients visiting them.

This overlap between the casual and sex-work networks probably facilitates the expansion of the HIV epidemic through the intermixing of the sexual networks.

Action taken: These venues with overlapping networks received the highest priority for interventions. FCT and Lagos had high proportions of venues facilitating both FSW and casual sexual networking, so interventions were targeted to these locations.

KF8: Focusing on specific geographic zones in a state containing a high proportion of the high-risk venues can maximise intervention coverage.

In each state, certain geographic zones contained a high proportion of high-risk venues.

In Gombe state, for example, EA results showed that $73 \%$ of patrons seeking casual sexual partners were visiting venues in zones 2 and 4.

Action taken: States targeted the specific zones with high densities of venues facilitating sexual networking and large proportions of patrons to maximise coverage.

KF9: Overall, only $18 \%$ of the high-risk venues had condoms available on site.

Action taken: Condom programming and distribution in the eight states has been scaled up in high-risk venues in the states.

\section{Programming for the rural general population}

KF10: FSWs are widespread in rural areas.

A relatively high proportion of villages in the eight states had FSWs living and working in them. This varied substantially across the states, with $40 \%$ of Benue villages having FSWs within them, whereas in Nassarawa, $23 \%$ of villages had FSWs within them.

Action taken: States are developing strategies for reaching these FSWs, particularly in Benue, where FSWs are widely distributed in rural areas, with relatively large numbers of FSWs overall.

KF11: Programmes are not always focusing on both reducing the number of partners and increasing condom use.

Sexual behaviour patterns varied substantially between states. Overall, a high proportion of both married and unmarried men and women reported having multiple sexual partners within the past six months (NACA 2013). Reported condom use tended to be low amongst both men and women: less than $50 \%$ in the last encounter with a casual sexual partner in all eight states (NACA 2013)

Action taken: In the eight states, programme strategies for rural areas were refocused on promoting partner reduction and condom use to reach key rural areas and to expand access to condoms.

\section{Practical implications}

The EA findings provided focused and targeted direction for planning, implementing and co-ordinating MARP interventions in Nigeria.

The findings informed the scale-up of interventions for nonbrothel-based FSWs in addition to interventions for brothelbased FSWs.

In 2014, the three main donors for the key population programmes (USAID, Global Fund and World Bank) funded the implementation of prevention interventions for both brothel-based and non-brothel-based FSWs in the key LGAs in the eight states.

Subsequently, refocused programming from the EA evidence has been incorporated into national guidelines.

In 2014, strategies developed from EA findings were adapted into the national FSW implementation guidelines (the approved standards for implementing FSW programmes in Nigeria).

The NACA is scaling up the conducting of EAs to cover the remaining 29 states in Nigeria.

With nationwide HIV prevention intervention for key populations being refocused for maximal coverage in the 
future, Nigeria is on track to achieving a national-level impact on the HIV epidemic.

The shift to implementing key population interventions only in key LGAs in the eight states has led to cost-efficiencies in resource allocation.

The EA findings provided important evidence for states to be able to reallocate their limited funds to high-density/highrisk areas. For example, resources have been committed for the MARP programming covering $70 \%-80 \%$ of FSWs in the key LGAs in the eight states from 2014 to 2016.

Focusing implementation on the profiled venues in key zones has allowed states to use resources available for general population interventions in a more focused way rather than being spread thinly across an entire state.

In most countries, there are insufficient resources available to ensure substantial coverage of all high-risk members of the general population. By redirecting these same funds to interventions in the profiled venues, states can reach a large segment of the general population seeking casual sex in urban areas.

The EA, a methodology used by countries and states to understand epidemic drivers and typologies, has played a critical rolein generating evidence for planning, implementing and co-ordinating HIV-prevention interventions in Nigeria. More specifically, it has informed the adoption of strategies to optimise coverage of HIV-prevention services for the MARPs as well as to improve the cost efficiency of the MARP interventions. EA results have also served to inform strategic direction regarding providing interventions for high-risk men and women within the general population.

Nigeria successfully adapted the EA to a mixed HIV epidemic type. The EAs that Nigeria conducted in the eight states were critical to generating evidence that would guide HIVprevention programmes beyond that obtained from periodic surveys such as HIV sentinel surveys amongst antenatal care attendees, NARHS and IBBSS. Countries faced with similar challenges of optimising prevention programming in a mixed HIV epidemic may find it useful to conduct such an EA.

\section{Limitations of the study}

The EA is designed to gather information rapidly from a large geographic area. It therefore only provides estimates of populations rather than actual numbers. EAs are therefore indicative rather than definitive. Another limitation of the mapping method is that it focuses on the most visible FSWs and other MARPs. Although this is likely to identify the key locations and populations of FSWs and IDUs, it is less effective at identifying networks of high-risk MSMs due to their highly stigmatised situation and discreet ways of interacting. Because the EA methodology is venue-based, members of the MARPs who do not gather in public spaces are likely to be under-represented.
The EA was adapted for use in Nigeria from the methods followed in India and other countries. A key change was adding the venue profiling as well as the rural appraisal components. Evidence of the impact of prevention programmes targeting high-risk members of the general population at high coverage levels has been validated only from the impact evaluation carried out on India's programme. The rapid nature of the EA also means that it can provide only limited insight into sexual behaviours and should thus be seen as a way of highlighting areas which could merit more in-depth social and behavioural research.

\section{Recommendations}

The mapping methodology for size estimation of MSMs needs to be reviewed to make it more effective at identifying networks of high-risk MSMs and other MARP members who do not gather in public spaces.

Both outcome and impact evaluations should be planned and funded as soon as possible for conducting them at the appropriate timing. They will contribute to the body of evidence on the ability of EAs to produce findings that are immediately relevant to prevention programming that will eventually improve Nigeria's mixed epidemic by reducing HIV transmission.

\section{Conclusion}

The EAs in eight Nigerian states filled critical gaps in the availableevidence on the Nigerian HIV epidemic and provided targeted directions for the effective planning, implementation and co-ordination of prevention interventions for key populations. Now that refocused prevention interventions are being implemented to maximise coverage and improve cost efficiencies by focusing on high-density MARP areas, Nigeria can expect to achieve the desired coverage of $70 \%-$ $80 \%$, the level needed to have an impact on HIV.

The EA findings have also contributed useful information regarding high-risk members of the general population through the venue profiles for casual sexual networking. The findings are being used to strengthen interventions for general populations seeking casual sex in urban areas as well as for networks which overlap with FSWs.

The EA is a methodology that other countries may want to replicate in order to identify more specific information that could guide HIV-prevention programming. The experience in Nigeria has demonstrated that the methodology can be adapted to the country or sub-country levels for a mixed HIV epidemic and can be used quickly to refocus prevention interventions and maximise coverage and the eventual impact.

\section{Acknowledgements}

The EAs in the first eight states in Nigeria were conducted by each of the State Agencies for the Control of AIDS with 
technical and financial support from the World Bank and NACA. The authors thank the SACA Programme Managers of the eight states where the EAs were conducted and the NACA Impact Evaluation Core team, who worked tirelessly during the entire process of the EAs. We also thank Dr Susan Griffey for the helpful mentorship and editorial assistance she provided during the revision of this article.

\section{Competing interests}

The authors declare that they have no financial or personal relationship(s) that may have inappropriately influenced them in writing this article.

\section{Authors' contributions}

I.E. (National Agency for the Control of AIDS) was the lead programme evaluation officer who co-ordinated the impact evaluation core team during the planning, field work, analysis and report writing process of the EAs. She was also the lead writer for this evaluation-use article. U.D (National Agency for the Control of AIDS) was a member of the impact evaluation core team and was involved in the planning, field work, analysis and report-writing process of the EAs. She also reviewed the article. K.O. (Director, Strategic Knowledge Department, NACA) was the leader of the impact evaluation core team and provided strategic oversight throughout the entire process of the EA. He contributed to the review of the article. J.B. (University of Manitoba) provided the technical support for the EA adaptation during the development of the operational manual for mapping, venue of profile and rural appraisal in Nigeria. O.E. (Director, Research and Evaluation Division (SFH)) was the lead person co-ordinating the involvement of SFH staff partnering with SACA during the planning, field work, analysis and report-writing process of the EAs. He also reviewed the article.

\section{References}

Blanchard, J., 2012, 'Heterogeneity of epidemics and implications for program planning', viewed 01 April 2015, from http://www.cdc.gov/stdconference/2012/ programscience/blanchardprogramsciencenationalstd2012.pdf

Emmanuel, F., Thompson, L.H., Salim, M., Akhtar, N., Reza, T.E., Hafeez, H. et al., 2013, 'The size and distribution of key populations at greater risk of HIV in Pakistan: Implications for resource allocation for scaling up HIV prevention programmes', Sexually Transmitted Infections 89, ii11-7. PMID: 23843454, http://dx.doi. org/10.1136/sextrans-2013-051017

Emmanuel, F., Thompson, L.H., Shajy, I., Parkash, R. \& Blanchard, J., 2012, Mapping Operational manual for Nigeria, University of Manitoba, Winnipeg.

Federal Ministry of Health (FMOH), [2007] 2010, 'HIV integrated biological and behavioural surveillance survey', viewed 17 February 2015, from http://www. popcouncil.org/pdfs/2011HIV_IBBSS2010.pdf.

Federal Ministry of Health (FMOH), [2008] 2010, 'National HIV sero-prevalence sentinel survey among ANC attendees', viewed 17 February 2015, from http:// www.nigeria-aids.org.

India Health Action Trust, 2010, 'HIV/AIDS situation and response in Karnakata: Epidemiological appraisal using data triangulation', viewed 30 March 2015, from http://www.ihat.in.

National Agency for the Control of AIDS (NACA), 2012, 'Global AIDS response: Country progress report', viewed 15 March 2015, from http://www.escr-net.org/ node/364991.

National Agency for the Control of AIDS (NACA), 2013, 'HIV epidemic appraisals in Nigeria: Evidence for prevention programme planning and implementation. Data from the first eight states', viewed 15 March 2015, from http://www.sbccvch. naca.gov.ng/sites/default/files/Local\%20Epidemic\%20Appraisal_glossy $\% 20$ report.pdf.

National Agency for the Control of AIDS (NACA), 2014, 'National guidelines for implementation of HIV prevention programmes for female sex workers in Nigeria', viewed 18 March 2015, from http://www.sbccvch.naca.gov.ng.

Shajy, I., Momah, A., Ameyan, W., Madu, B. \& Blanchard, J., 2012a, Rural appraisal Operational manual for Nigeria, University of Manitoba, Winnipeg.

Shajy, I., Momah, A., Ameyan, W., Madu, B. \& Blanchard, J., 2012b, Venue profiling Operational manual for Nigeria, University of Manitoba, Winnipeg.

UNAIDS Reference Group on Estimates, Modeling and Projections, 2013, 'Identifying populations at greatest risk of infection - Geographic hotspots and key populations', viewed 30 March 2015, from http://www.epidem.org/ resources.

Wilson, D. \& Halperin, D.T., 2008, “"Know your epidemic, know your response”: A useful approach, if we get it right', The Lancet 372, 423-426. PMID: 18687462 , $\mathrm{http}: / / d x$.doi.org/10.1016/S0140-6736(08)60883-1 